\title{
On the Harsanyi payoff vectors and Harsanyi imputations
}

\author{
Jean Derks • Gerard van der Laan • \\ Valery Vasil'ev
}

Published online: 16 November 2008

(C) The Author(s) 2008. This article is published with open access at Springerlink.com

\begin{abstract}
This article discusses the set of Harsanyi payoff vectors of a cooperative TU-game, also known as the Selectope. We reconsider some results on Harsanyi payoff vectors within a more general framework. First, an intuitive approach is used, showing that the set of Harsanyi payoff vectors is the core of an associated convex game. Next, the set of individual rational Harsanyi payoff vectors, the Harsanyi imputations in short, is considered. Existence conditions are provided, and if non-empty, we provide a description as the core of a well-defined convex game, and show that it is an externally stable set.
\end{abstract}

Keywords TU-games · Convex games · Core · Harsanyi set · Imputations · External stability

\section{JEL Classification C71}

\footnotetext{
J. Derks $(\varangle)$

Faculty of Humanities and Sciences, University of Maastricht, P.O. Box 616, 6200 MD Maastricht, The Netherlands

e-mail: jean.derks@micc.unimaas.nl

G. van der Laan

Department of Econometrics and Tinbergen Institute, Vrije Universiteit, De Boelelaan 1105,

1081 HV Amsterdam, The Netherlands

e-mail: glaan@econ.vu.nl

V. Vasil'ev

Sobolev Institute of Mathematics, Prosp. Koptyuga 4, 630090 Novosibirsk, Russia

e-mail: vasilev@math.nsc.ru
} 


\section{Introduction}

A cooperative game with transferable utilities, or simply a game, describes a situation in which groups of players, the coalitions, can obtain certain payoffs by cooperation. A solution is a mapping which assigns to every game a set of payoff distributions over the players in the game. Well-known set-valued solutions are the Core and the set of all random order values, also known as the Weber set. In this article these sets are considered in the context of the set of payoff vectors obtained by all possible distributions of the Harsanyi dividends (Harsanyi 1959, 1963), of the coalitions among its members. We call this set the Harsanyi set; it is considered first in Hammer et al. (1997) (as the Selectope) and, independently, in Vasil'ev (1978, in Russian, and 1981). These articles show that the Harsanyi set encloses the core of the game, and Vasil'ev furthermore proved that this set has a core-type structure, a result that is shown independently in Derks et al. (2000).

In Hammer et al. (1997) the inclusion of the core is shown with the help of a network flow model, whereas Vasil'ev (1978) applies induction techniques. Here, we will apply a transparent approach, related to the ones in Vasil'ev (1981) and Derks et al. (2000), based on a convenient adaptation of the game into a convex game. In this way, and with the help of the characterization of the extreme points of the core of convex games in Shapley (1971), not only the inclusion of the core in the Harsanyi set is proved but also its core-type structure is revealed. Relations between the Harsanyi set and the Weber set are discussed in Derks et al. (2006).

Here, we will provide new and intuitive proofs for the results mentioned above, and we reposition several others within a more general framework. Starting with the preliminary Sect.2, the game theoretic notions are further discussed in Sect. 3. We provide a new proof for the characterization of the Harsanyi set as the core of an adapted, convex game. In Sect. 4 we generalize several results, known for the set of the so-called Harsanyi imputations, being the individually rational payoff vectors in the Harsanyi set. We discuss necessary and sufficient conditions for the existence of these imputations, and show that the Harsanyi Imputation set, like the Harsanyi set, is the core of an adapted, convex game. In the concluding Sect. 5, we provide a class of externally stable sets. The Harsanyi imputation set, if non-empty, is one of them, implying its external stability.

\section{Preliminaries}

A cooperative game with transferable utilities, or simply a game, is a pair $(N, v)$, where $N=\{1, \ldots, n\}$ is a finite set of players, and $v: 2^{N} \rightarrow \mathbb{R}$ such that $v(\emptyset)=0$, is the characteristic function yielding for each subset $S$ of $N$ the payoff $v(S)$ that can be achieved if the players in $S$ cooperate. Non-empty subsets of the player set are, therefore, called coalitions. We denote the collection of all non-empty subsets of $N$ by $\Omega=\{S \subseteq N: S \neq \emptyset\}$. A payoff vector is a vector $x \in \mathbb{R}^{n}$ assigning payoff $x_{i} \in \mathbb{R}$ to player $i \in N$. For a payoff vector $x \in \mathbb{R}^{n}$ and $S \in \Omega$, we denote with $x(S)=\sum_{i \in S} x_{i}$ the total payoff to the players in coalition $S$. 
In a game $(N, v)$, or $v$ for short, the main issue is the distribution of the worth $v(N)$ of the grand coalition among the players. A payoff vector $x$ is therefore said to be efficient if the total payoff $x(N)$ equals $v(N)$; it is said to be individually rational if each player $i \in N$ gets at least his own worth $v(\{i\})$. A payoff vector is called an imputation if it is both efficient and individually rational; the set of imputations of the game $v$ is denoted by $I(v)$ :

$$
I(v)=\left\{x \in \mathbb{R}^{n}: x(N)=v(N), \quad x_{i} \geq v(\{i\}), i \in N\right\} .
$$

One may consider the elements of the imputation set as those distributions of the grand coalition worth that 'meet the demands' of the single players. An efficient payoff vector $x$ that satisfies $x(S) \geq v(S)$ for each coalition $S$ is called stable for obvious reasons. The set of stable payoff vectors is called the core of the game $v$ and is denoted by $C(v)$ :

$$
C(v)=\left\{x \in \mathbb{R}^{n}: x(N)=v(N), x(S) \geq v(S), S \in \Omega\right\} .
$$

Unfortunately, the core, and also the imputation set, may be empty.

Let $\Pi(N)$ (or $\Pi$ ) denote the set of all permutations $\pi: N \rightarrow N$ on the player set $N$. For a permutation $\pi \in \Pi$, assigning rank number $\pi(i) \in\{1,2, \ldots, n\}=N$ to player $i \in N$, define the set $\pi^{i}$ to be $\{j \in N: \pi(j) \leq \pi(i)\}$; it denotes the set of all players with rank number at most equal to the rank number of $i$, including $i$. Then the marginal contribution vector $m^{\pi}(v) \in \mathbb{R}^{n}$ of game $v$ and permutation $\pi$ is given by

$$
m_{i}^{\pi}(v)=v\left(\pi^{i}\right)-v\left(\pi^{i} \backslash\{i\}\right), \quad i \in N,
$$

and thus assigns to player $i$ its marginal contribution to the worth of the coalition consisting of all his predecessors in $\pi$.

The well-known Shapley value (Shapley 1953), has been characterized as being the average of the marginal contribution vectors over all permutations. It is an element of the convex hull of the marginal contribution vectors of $v$, denoted by $W(v)$, and referred to as the Weber set. Contrary to the core, the Weber set is always non-empty. It contains the core as a subset, as shown by Weber (1988); it may, however, have no points in common with the imputation set (see Martinez de Albéniz and Rafels 1998); of course this only occurs when the core is empty.

The core and the Weber set coincide if and only if the game $v$ fulfills the inequalities $v(S \cup T)+v(S \cap T) \geq v(S)+v(T)$ for every pair of coalitions $S, T \in \Omega$ (Shapley 1971; Ichiishi 1981). A game is called convex when these inequalities are fulfilled.

The dividends $\Delta^{S}(v), S \in \Omega$, of the game $v$ follow recursively from the system of equations

$$
v(S)=\sum_{T \subseteq S} \Delta^{T}(v), \quad S \in \Omega
$$

The dividend is first discussed in Harsanyi $(1959,1963)$ as a notion that captures the value of a coalition which is solely acquired by the cooperation of all players 
within the coalition. Here, we will consider payoff vectors obtained by distributing the dividend of each coalition $S$ over the players in $S$. To facilitate this distribution we make use of the weight systems $p=\left(p_{i}^{S}\right)_{S \in \Omega, i \in S}$, assigning to each coalition $S$ and member $i$ of $S$ a weight $p_{i}^{S}$. A weight system $p$ is called a sharing system if all weights are non-negative, and the weights $p_{i}^{S}, i \in S$, sum up to 1 for each coalition $S$; the collection of sharing systems is thus given by

$$
P=\left\{p=\left(p_{i}^{S}\right)_{S \in \Omega, i \in S}: p \geq 0, \sum_{j \in S} p_{j}^{S}=1, \text { for each } S \in \Omega\right\}
$$

For a game $v$ and sharing system $p \in P$, let the payoff vector $\phi^{p}(v) \in \mathbb{R}^{n}$ be given by

$$
\phi_{i}^{p}(v)=\sum_{S: i \in S} p_{i}^{S} \Delta^{S}(v), \quad i \in N
$$

i.e., the payoff $\phi_{i}^{p}(v)$ to player $i$ is the sum, over all coalitions $S$ containing $i$, of the share $p_{i}^{S}$ of player $i$ in the Harsanyi dividend $\Delta^{S}(v)$ of coalition $S$. We therefore call the payoff vector $\phi^{p}(v)$ a Harsanyi payoff vector. Observe that, due to the equality $v(N)=\sum_{S \in \Omega} \Delta^{S}(v)$, for each sharing system $p$ it holds that $\sum_{i \in N} \phi_{i}^{p}(v)=v(N)$, and thus each Harsanyi payoff vector is efficient. Examples of Harsanyi payoff vectors are the marginal contribution vectors (Derks et al. 2006), and in particular, the Shapley value which is the Harsanyi payoff vector with weights $p_{i}^{S}=(|S|)^{-1}, i \in S, S \in \Omega$.

Let $H(v)$ denote the set of all Harsanyi payoff vectors of the game $v$, i.e.,

$$
H(v)=\left\{\phi^{p}(v): p \in P\right\} .
$$

This set is introduced as the so-called Selectope in Hammer et al. (1997). Independently, Harsanyi payoff vectors and the set $H(v)$ are discussed in Vasil'ev (1978, 1981). Here, we prefer to call the set $H(v)$ the Harsanyi set instead of Selectope, because we want to stress the property of distributing the Harsanyi dividends instead of the role of the selectors as discussed in Derks et al. (2000).

\section{The Harsanyi set}

In this section, we will recall some results on the Harsanyi set and reposition them in the historical context, and provide new proofs. These results concern the relationship between the core and the Harsanyi set, and the geometrical structure of the Harsanyi set. In particular, we provide alternative proofs for the Harsanyi set enclosing the core, and having a core-type structure.

For a game $v$ we consider the following game $v_{H}$, defined by

$$
v_{H}(S)=\min _{x \in H(v)} x(S) \quad S \in \Omega
$$


The game $v_{H}$ is called the Harsanyi mingame; it specifies the minimal amount the coalitions attain in a Harsanyi payoff vector. All these vectors are efficient in the game $v$. Therefore, $v_{H}(N)=v(N)$, and we may conclude that the Harsanyi set is a subset of the core of $v_{H}$ :

$$
H(v) \subseteq C\left(v_{H}\right)
$$

By definition, the Harsanyi payoff vectors distribute the dividends $\Delta^{S}(v)$ among the members of coalition $S \in \Omega$, so that for a given coalition $S$ the dividend $\Delta^{T}(v)$ is fully allocated to the members of $S$ if $T \subseteq S$, and fully allocated to the players outside $S$ if $T \cap S=\emptyset$; otherwise, i.e., if $T \cap S \neq \emptyset$ and $T \backslash S \neq \emptyset$, the amount $\Delta^{T}(v)$, when distributed among the players in $T$, may be allocated in full, part, or not to players in $S$. The total distribution in a Harsanyi payoff vector to players in coalition $S$ is therefore minimized when the distribution of $\Delta^{T}(v)$, with $T$ such that $T \cap S \neq \emptyset$ and $T \backslash S \neq \emptyset$ holds, is only performed among the members of $S$ in case $\Delta^{T}(v)<0$, and among the players outside $S$ otherwise. This shows that

$$
v_{H}(S) \geq \sum_{T \subseteq S} \Delta^{T}(v)+\sum_{T: T \cap S \neq \emptyset, T \backslash S \neq \emptyset, \Delta^{T}(v)<0} \Delta^{T}(v), \quad S \in \Omega .
$$

Actually, equality holds in (2). To show this consider for a permutation $\pi$ of the player set $N=\{1,2, \ldots, n\}$ the following payoff vector

$$
x_{i}^{\pi}=\sum_{S \subseteq \pi^{i}, i \in S, \Delta} \Delta_{(v)>0} \Delta^{S}(v)+\sum_{S \subseteq \pi^{-i}, i \in S, \Delta^{S}(v)<0} \Delta^{S}(v), \quad i \in N,
$$

with $\pi^{i}$ being the already defined set of predecessors of $i$, and $\pi^{-i}=\{j \in N$ : $\pi(j) \geq \pi(i)\}$ the set of successors of $i$ in $\pi$. It is straightforward that $x^{\pi}$ is a Harsanyi payoff vector in the game $v$ as each dividend $\Delta^{T}(v)$ of $v$ is distributed to one of the players in $T$. In other words, the vector $x^{\pi}$ assigns to each player $i$ all positive dividends $\Delta^{T}(v)$ of coalitions $T$ where $i$ is the $\pi$-last member, and all negative dividends of coalitions where player $i$ is the $\pi$-first member. The coalition of predecessors of $i$ therefore attains all dividends, namely, $\Delta^{T}(v), T \subseteq \pi^{i}$, and the negative dividends $\Delta^{T}(v)$ of coalitions $T$ with $T \cap \pi^{i} \neq \emptyset$ and $T \backslash \pi^{i} \neq \emptyset$.

For any coalition $S$ there is a permutation $\pi$ and player $i$ such that $S$ consists precisely of the predecessors of $i$. We thus have

$$
v_{H}(S) \leq x^{\pi}(S)=\sum_{T \subseteq S} \Delta^{T}(v)+\sum_{T: T \cap S \neq \emptyset, T \backslash S \neq \emptyset, \Delta^{T}(v)<0} \Delta^{T}(v), \quad S \in \Omega,
$$

so that equality in (2) follows.

We just showed that $v_{H}\left(\pi^{i}\right)=x^{\pi}\left(\pi^{i}\right)$ for any permutation $\pi$ and player $i$; so, the payoff $x_{i}^{\pi}$ to player $i$ equals $v_{H}\left(\pi^{i}\right)-v_{H}\left(\pi^{i} \backslash\{i\}\right)$, which is actually the payoff to player $i$ in the marginal contribution vector of $v_{H}$ corresponding to permutation $\pi$. This implies that the marginal contribution vectors of $v_{H}$ are core elements of 
$v_{H}$. The Weber set $W\left(v_{H}\right)$ is contained in the core of $v_{H}$, so $W\left(v_{H}\right)=C\left(v_{H}\right)$ must hold. Therefore, we conclude that $v_{H}$ is a convex game. Furthermore, as convex combinations of Harsanyi payoff vectors are again Harsanyi payoff vectors, we have $C\left(v_{H}\right)=W\left(v_{H}\right) \subseteq H(v)$, and with (1) equality follows.

Theorem 1 (Vasil'ev 1981; Derks et al. 2000) For each game v, the Harsanyi mingame $v_{H}$ is convex, and $H(v)=C\left(v_{H}\right)$.

The Harsanyi set $H(v)$ of a game $v$ has therefore a core-type structure, being the core $C\left(v_{H}\right)$ of the corresponding Harsanyi mingame $v_{H}$. Since the game $v$ majorizes its Harsanyi mingame, with equal value for the grand coalition $N$, the core of $v$ must therefore be a subset of the core of $v_{H}$.

Corollary 1 (Hammer et al. 1997; Vasil'ev 1981) For each game the stable payoff vectors are Harsanyi payoff vectors.

\section{Harsanyi imputations}

It is desirable that a payoff vector is efficient and, if possible, also individually rational. Martinez de Albéniz and Rafels (1998) show that the Weber set may not contain imputations. This is also the case for the larger Harsanyi set, as the following example shows.

Let $N$ be a player set with four or more players, and $S$ a coalition with 2 players. Consider the game $(N, v)$ where the dividends of all coalitions are 0 except for the coalitions $S$ and $N \backslash S: \Delta^{S}(v)=1$, and $\Delta^{N \backslash S}(v)=-1$. Then $v(N)=0$ and $v(T)=1$ for coalitions $T \neq N$ with $S \subseteq T$, and $v(T)=-1$ for coalitions $T \neq N$ with $N \backslash S \subseteq T$, and $v(T)=0$ otherwise. The game has only one imputation $x: x_{i}=0$ for each player $i$. Now, using the inequality (2) we obtain $v_{H}(S) \geq 1$, and knowing that each Harsanyi payoff vector should distribute at least $v_{H}(S)$ to the players in $S$, the imputation $x$ cannot be a Harsanyi payoff vector. So, the nonempty imputation set of $v$ has no elements in common with its Harsanyi set.

Let $H I(v)$ denote the intersection of the Harsanyi set and the imputation set, i.e.,

$$
H I(v)=\left\{x \in H(v): x_{i} \geq v(\{i\}), i \in N\right\}=\left\{x \in C\left(v_{H}\right): x_{i} \geq v(\{i\}), i \in N\right\} .
$$

We call its elements Harsanyi imputations.

Let $w$ be a game on player set $N$, and $z \in \mathbb{R}^{n}$ an arbitrarily chosen vector. We denote the portion of the core of $w$ that lies above $z$ by $C^{z}(w)$, i.e.,

$$
C^{z}(w)=\{x \in C(w): x \geq z\}
$$

Clearly, we have $H I(v)=C\left(v_{H}\right) \cap I(v)=C^{z}(w)$, with $w=v_{H}$ and $z_{i}=v(\{i\})$, $i \in N$. We show that $C^{z}(w)$ is either empty, or is equal to the core of the game $w^{z}$ defined by

$$
w^{z}(S)=\max _{T \subseteq S}\{w(T)+z(S \backslash T)\}, \quad S \in \Omega .
$$


Intuitively, the payoffs $z_{i}, i \in N$, may be considered here as a kind of minimum participation level of the players, and in this context the game $w^{z}$ is observed when the players are offered to choose between cooperation in the game $w$ or to be paid according to the (not necessarily efficient) payoff vector $z$. In this situation the elements of $C^{z}(w)$ are the preferred outcomes.

The following theorem shows that in case these outcomes exist they are exactly the stable payoff vectors of the game $w^{z}$.

Theorem 2 Let $w$ be a game on player set $N$ and $z \in \mathbb{R}^{n}$. If $w(N) \geq w(T)+z(N \backslash T)$ for each coalition $T$, then $C^{z}(w)=C\left(w^{z}\right)$; otherwise, $C^{z}(w)=\emptyset$.

Proof First, consider the case that a coalition $T$ exists, with $w(N)<w(T)+z(N \backslash T)$. Then for each vector $y \in \mathbb{R}^{n}$ satisfying $y \geq z$ and $y(T) \geq w(T)$ it holds that $y(N)=y(T)+y(N \backslash T) \geq w(T)+z(N \backslash T)>w(N)$, so that $C^{z}(w)$ is empty.

Secondly, let $w(N) \geq w(T)+z(N \backslash T)$ for all $T \subseteq N$. Then it follows that

$$
w^{z}(N)=\max _{T \subseteq N}\{w(T)+z(N \backslash T)\}=w(N) .
$$

Now, suppose that $y \in C^{z}(w)$. Then we have, for each coalition $S$,

$$
y(S)=y(T)+y(S \backslash T) \geq w(T)+z(S \backslash T), \quad \text { for all } T \subseteq S,
$$

and thus $y(S) \geq w^{z}(S)$. This shows that $y \in C\left(w^{z}\right)$. On the other hand, for each $y \in C\left(w^{z}\right)$ we have

$$
y(S) \geq w^{z}(S) \geq \max \{w(S), z(S)\}, \quad S \in \Omega .
$$

Together with $y(N)=w^{z}(N)=w(N)$ this proves that $y \in C(w)$ and $y \geq z$, i.e., $y \in C^{z}(w)$. Hence $C^{z}(w)=C\left(w^{z}\right)$.

The proof shows that the equality $w(N)=w^{z}(N)$ implies $C^{z}(w)=C\left(w^{z}\right)$. It should be noted that $w(N)=w^{z}(N)$ does not imply that $C^{z}(w)$ is non-empty. For example, let $w$ be a monotonic game with an empty core (for instance, $w(S)=1$ for all non-empty coalitions $S$ ), and $z=0$. Then $w^{z}=w$, implying $w(N)=w^{z}(N)$. However, $C^{z}(w)=C\left(w^{z}\right)=C(w)=\emptyset$.

It is interesting to examine which of the properties of $w$ are invariant under the transformation into $w^{z}$. In particular, the convexity property is of interest in our context, since we want to apply the previous theorem on the Harsanyi mingame $v_{H}$, which is a convex game. Actually, we have the following theorem.

Theorem 3 Let $w$ be a convex game on player set $N$ and $z \in \mathbb{R}^{n}$. Then $w^{z}$ is also convex.

Proof Let $S, T$ be two arbitrary non-empty coalitions. Then there exist coalitions $U \subseteq$ $S$ and $V \subseteq T$ such that $w^{z}(S)=w(U)+z(S \backslash U)$ and $w^{z}(T)=w(V)+z(T \backslash V)$. By subtracting the equality $z(U)+z(V)=z(U \cup V)+z(U \cap V)$ from the equality $z(S)+z(T)=z(S \cup T)+z(S \cap T)$ we obtain

$$
z(S)-z(U)+z(T)-z(V)=z(S \cup T)-z(U \cup V)+z(S \cap T)-z(U \cap V) .
$$


Taking into account that $U \subseteq S$ and $V \subseteq T$, this equality reduces to

$$
z(S \backslash U)+z(T \backslash V)=z((S \cup T) \backslash(U \cup V))+z((S \cap T) \backslash(U \cap V))
$$

Therefore,

$$
\begin{aligned}
w^{z}(S)+w^{z}(T)= & w(U)+z(S \backslash U)+w(V)+z(T \backslash V) \\
\leq & w(U \cup V)+w(U \cap V)+z((S \cup T) \backslash(U \cup V)) \\
& +z((S \cap T) \backslash(U \cap V)) \\
\leq & w^{z}(S \cup T)+w^{z}(S \cap T),
\end{aligned}
$$

thus implying the convexity of $w^{z}$.

Now, consider the convex Harsanyi mingame $v_{H}$ and take $z \in \mathbb{R}^{n}$ with $z_{i}=v(\{i\})$, $i \in N$. Then $\left(v_{H}\right)^{z}$ is convex and thus $C\left(\left(v_{H}\right)^{z}\right)$ is non-empty. Applying Theorem 2 with $w=v_{H}$ and $z_{i}=v(\{i\}), i \in N$, we obtain the following result.

Corollary 2 (Vasil'ev 1981) Harsanyi imputations of a game $v$ exist if and only if for all coalitions $T$ we have $v(N) \geq v_{H}(T)+\sum_{i \notin T} v(\{i\})$. If non-empty, the set of Harsanyi imputations is equal to the core of the convex game $\left(v_{H}\right)^{z}$, with $z_{i}=v(\{i\})$, $i \in N$.

Martinez de Albéniz and Rafels (1998) show that the Weber set of a game $v$ has a non-empty intersection with the set of imputations if $v$ fulfills the very mild condition $v(N) \geq v(T)+\sum_{i \notin T} v(\{i\})$ for all coalitions $T$. The corollary shows that the set of Harsanyi imputations is non-empty if and only if the game $v$ fulfills the weaker collection of inequalities $v(N) \geq v_{H}(T)+\sum_{i \notin T} v(\{i\}), T \in \Omega$. Observe that in the example at the start of this section we have $0=v(N)<1=v_{H}(S)+\sum_{i \notin S} v(\{i\})$.

\section{Harsanyi imputations and external stability}

Rafels and Tijs (1997) show that the Weber set is externally stable. Here, a set $G \subseteq I(v)$ is called externally stable in a game $v$ if for each imputation $x$ outside $G$ there is an imputation $y$ in $G$ and a coalition $S$ such that $x_{i}<y_{i}$ for each player $i$ in $S$, and $y(S) \leq v(S)$ (Von Neumann and Morgenstern 1944).

It is shown in Vasil'ev (1988) that the set $H I(v)$ of Harsanyi imputations is externally stable. Its proof involves some complex techniques. Here, we provide a more accessible proof for this stability result.

We first prove a more general external stability result in which the notion of a large core plays a central role. We say that a game $v$ has a large core if for each vector $x \in \mathbb{R}^{n}$ with $x(S) \geq v(S)$ for each coalition $S$, there is a vector $y \in C(v)$ such that $y \leq x$. Convex games are known to have a large core (Sharkey 1982).

Theorem 4 For each game $v$ and convex game $v^{\prime}$ with $v^{\prime} \leq v$, the intersection $C\left(v^{\prime}\right) \cap I(v)$ is either empty or externally stable (w.r.t. $\left.v\right)$. 
Proof Observe that we only need to consider the case $C\left(v^{\prime}\right) \cap I(v) \neq \emptyset$ and $I(v) \backslash C\left(v^{\prime}\right)$ $\neq \emptyset$. According to Theorem 2 we have

$$
C\left(v^{\prime}\right) \cap I(v)=C\left(v^{\prime \prime}\right)
$$

where $v^{\prime \prime}=\left(v^{\prime}\right)^{z}$, with $z_{i}=v(\{i\}), i \in N$, i.e.,

$$
v^{\prime \prime}(S)=\max _{T \subseteq S}\left\{v^{\prime}(T)+\sum_{i \in S \backslash T} v(\{i\})\right\}, \quad S \subseteq N
$$

Take an arbitrary element $y$ of $I(v) \backslash C\left(v^{\prime}\right)$. To prove the theorem, we construct an imputation $y^{\prime}$ of $v$ in $C\left(v^{\prime}\right) \cap I(v)$ that dominates $y$.

Since $y \notin C\left(v^{\prime}\right)$, there is at least one coalition $S$ with $y(S)<v^{\prime}(S)$ and thus also $y(S)<v^{\prime \prime}(S)$, since $v^{\prime} \leq v^{\prime \prime}$. Choose a coalition $\widehat{S}$ such that $y(\widehat{S})<v^{\prime \prime}(\widehat{S})$ and $y(T) \geq v^{\prime \prime}(T)$ for each subcoalition $T$ of $\widehat{S}$. Notice that for each $T \subset \widehat{S}$,

$$
v^{\prime \prime}(\widehat{S})>y(\widehat{S})=y(T)+y(\widehat{S} \backslash T) \geq v^{\prime \prime}(T)+\sum_{i \in \widehat{S} \backslash T} v(\{i\}) \geq v^{\prime}(T)+\sum_{i \in \widehat{S} \backslash T} v(\{i\}),
$$

so that we actually must have $v^{\prime \prime}(\widehat{S})=v^{\prime}(\widehat{S})$, implying that

$$
v^{\prime \prime}(\widehat{S}) \leq v(\widehat{S}) .
$$

Now, increase all coordinates $y_{i}, i \in \widehat{S}$, by the same amount $|\widehat{S}|^{-1}\left(v^{\prime \prime}(\widehat{S})-y(\widehat{S})\right)$, and all other coordinates by a sufficiently large amount such that the payoff vector thus obtained, say $x$, majorizes $v^{\prime \prime}$, i.e., $v^{\prime \prime}(T) \leq x(T)$ for all $T \in \Omega$. Since $v^{\prime \prime}$ is convex because $v^{\prime}$ is convex, $v^{\prime \prime}$ has a large core. This implies the existence of a vector $y^{\prime} \in C\left(v^{\prime \prime}\right)$ such that $y^{\prime} \leq x$. Since

$$
v^{\prime \prime}(\widehat{S}) \leq y^{\prime}(\widehat{S}) \leq x(\widehat{S})=\sum_{i \in \widehat{S}}\left(y_{i}+\mid \widehat{S}^{-1}\left(v^{\prime \prime}(\widehat{S})-y(\widehat{S})\right)\right)=v^{\prime \prime}(\widehat{S})
$$

it follows that $v^{\prime \prime}(\widehat{S})=y^{\prime}(\widehat{S})=x(\widehat{S})$. With $y^{\prime} \leq x$ we conclude that $y_{i}^{\prime}=x_{i}$ for all $i \in \widehat{S}$, and thus $y_{i}^{\prime}=x_{i}>y_{i}$ for all $i \in \widehat{S}$. Combined with (3), it follows that $y^{\prime}$ dominates $y$ (w.r.t. $v$ ), proving that $C\left(v^{\prime}\right) \cap I(v)$ is an externally stable subset of $I(v)$.

To apply this theorem to the set of Harsanyi imputations, recall that $H I(v)=$ $C\left(v_{H}\right) \cap I(v)$, the Harsanyi mingame $v_{H}$ is convex, and $v_{H} \leq v$. We thus arrive at the following result.

Corollary 3 (Vasil'ev 1988) The set of Harsanyi imputations of a game is either empty or externally stable.

Together with Corollary 2 it follows that the set of Harsanyi imputations is externally stable if and only if $v(N) \geq v_{H}(T)+\sum_{i \notin T} v(\{i\})$ for all coalitions $T$. 
Observe further that, by Corollary 3, the Harsanyi imputation set should contain all non-dominated imputations. These imputations constitute the well-known Domination core. The core is always contained in the Domination core, but not vice versa in general; it may happen that the core is empty whereas the Domination core is non-empty. Therefore, Corollary 3 is a refinement of Corollary 1.

Acknowledgements This study was partly done whilst Valeri Vasil'ev was visiting the Department of Econometrics at the Free University, Amsterdam. Financial support from the Netherlands Organisation for Scientific Research (NWO) in the framework of the Russian-Dutch programme for scientific cooperation, is gratefully acknowledged. The third author would also like to acknowledge partial financial support from the Russian Fund of Basic Research (Grants 98-01-00664 and 00-15-98884) and the Russian Humanitarian Scientific Fund (Grant 02-02-00189a).

Open Access This article is distributed under the terms of the Creative Commons Attribution Noncommercial License which permits any noncommercial use, distribution, and reproduction in any medium, provided the original author(s) and source are credited.

\section{References}

Derks, J., Haller, H., \& Peters, H. (2000). The selectope for cooperative games. International Journal of Game Theory, 29, 23-38.

Derks, J., van der Laan, G., \& Vasil'ev, V. A. (2006). Characterizations of the random order values by Harsanyi payoff vectors. Mathematical Methods of Operations Research, 64, 155-163.

Hammer, P. L., Peled, U. N., \& Sorensen, S. (1977). Pseudo-boolean functions and game theory. I. Core elements and Shapley value. Cahiers du CERO, 19, 159-176.

Harsanyi, J. C. (1959). A bargaining model for cooperative $n$-person games. In A. W. Tucker, \& R. D. Luce (Eds.), Contributions to the theory of games IV (pp. 325-355). Princeton NJ: Princeton University Press.

Harsanyi, J. C. (1963). A simplified bargaining model for the $n$-person game. International Economic Review, 4, 194-220.

Ichiishi, T. (1981). Super-modularity: Applications to convex games and the greedy algorithm for LP. Journal of Economic Theory, 25, 283-286.

Martinez de Albéniz, F., \& Rafels, C. (1998). On the intersection between the imputation set and the weber set. Annals of Operations Research, 84,111-120.

Rafels, C., \& Tijs, S. H. (1997). On the cores of cooperative games and the stability of the weber set. International Journal of Game Theory, 26, 491-499.

Shapley, L. S. (1953). A value for $n$-person games. In H. W. Kuhn, \& A. W. Tucker (Eds.), Contributions to the theory of games II (pp. 307-317). Princeton NJ: Princeton University Press.

Shapley, L. S. (1971). Cores of convex games. International Journal of Game Theory, 1, 11-26.

Sharkey, W. W. (1982). Cooperative games with large cores. International Journal of Game Theory, 11, $175-182$.

Vasil'ev, V. A. (1978). Support function of the core of a convex game. Optimizacija, 21. 30-35 (in Russian).

Vasil'ev, V. A. (1981). On a class of imputations in cooperative games. Soviet Mathematics Doklady, 23, 53-57.

Vasil'ev, V. A. (1988). Characterization of the cores and generalized NM-solutions for some classes of cooperative games. In Proceedings of Institute of Mathematics, Novosibirsk, 'Nauka' (Vol. 10, pp. 63-89) (in Russian).

Vasil'ev, V. A., \& van der Laan, G. (2002). The Harsanyi set for cooperative TU-games. Siberian Advances in Mathematics, 12, 97-125.

Von Neumann, J., \& Morgenstern, O. (1944). Theory of games and economic behavior. Princeton, NJ: Princeton University Press.

Weber, R. J. (1988). Probabilistic values for games. In A.E. Roth (Ed.), The Shapley value, essays in honor of L.S. Shapley (pp. 101-119). Cambridge: Cambridge University Press. 